\title{
El docente escritor: una estrategia para la enseñanza de la expresión escrita
}

Anielka Patricia. CARBALLO PALMA Universidad Nacional Autónoma de Nicaragua, Managua anidocente@gmail.com https://idorcid.org/0000-0002-1388$295 X$

(c) UNAN-Managua Recibido: abril 2017 Aprobado: junio 2017

\section{The teacher writer: a strategy for teaching written expression}

\section{RESUMEN}

La enseñanza-aprendizaje de la expresión escrita ha suscitado una serie de modelos y enfoques que orientan al docente. Sin embargo, el alumno muchas veces no posee los conocimientos, habilidades y actitud para aprender a mejorar su redacción. La intervención del docente, como escritor experto, es esencial para que el estudiante lo observe y aprenda de él, teniendo siempre presente que el proceso de escritura es individual y el alumno debe buscar su propio estilo.

Palabras claves: expresión escrita, modelos y enfoques de escritura, docente, redacción, alumno.

\section{SUMMARY}

The teaching and learning of writing has raised a number of models and approaches that guide the teacher. However, students often do not have the knowledge, skills and attitude to learn to improve their writing. The intervention of the teacher as expert writer, is essential for the student to observe and learn from it, bearing in mind that the writing process is individual and the student must find his own style.

Keywords: writing, writing models and approaches, teaching, writing, student.

\begin{abstract}
"Maestra, ¿cómo inicio?", "No sé cómo escribirlo", "Todo lo tengo en la cabeza, pero cómo lo pongo en el papel". Estas son algunas de las expresiones que constantemente escuchamos los docentes al momento que solicitamos a nuestros alumnos escribir. Lamentablemente, muchas veces no sabemos otra respuesta más que: "Inténtelo", "Vamos, usted puede", "De eso se trata, sáquelo de la cabeza", "escriba con sus palabras lo que quiere decir". Sin embargo, estas palabras son insuficientes. El alumno tiene grandes dificultades al iniciar el proceso de escritura.
\end{abstract}

Este ensayo investigativo tiene como propósito fundamental reflexionar sobre la práctica educativa que adoptamos como docentes durante la enseñanza-aprendizaje de la expresión escrita. La reflexión estará acompañada por algunas referencias teóricas sobre la práctica docente más idónea para la enseñanza-aprendizaje de la expresión escrita. De igual forma, se irá ejemplificando con algunos trabajos realizados por los estudiantes de primer año de la carrera de Informática Educativa (2011) de la Facultad de Educación e Idiomas de la UNAN-Managua. 


\section{Algunos modelos y enfoques}

Empecemos señalando lo que algunos teóricos han manifestado sobre la enseñanza de la escritura. Partamos de lo que el alumno debe tener o dominar. Cassany (2002) ha considerado que la escritura requiere tanto de conocimientos como de habilidades, pero sobre todo de actitud. Siguiendo está lógica, el estudiante debe tener conocimientos esenciales sobre: Gramática y ortografía; coherencia y cohesión de los textos; recursos retóricos e información general. Sin embargo, esto no es suficiente, también debe reunir ciertas habilidades como: analizar, elaborar esquemas, buscar información, plantear ideas, relacionar textos, entre otras. Todos estos elementos son esenciales para la formación del escritor. La pregunta sería ¿cómo se conjugan en la mente del alumno al momento que se le pide escribir? La respuesta a esta cuestión es esencial para mejorar la actitud del educando ante la escritura.

Muchos de nuestros estudiantes se sienten agobiados al momento que se les pide escriban un texto, por muy pequeño que sea. Esto se debe muchas veces a que el dominio de los conocimientos y habilidades antes descritas por Cassany es endeble en ellos. Años estudiando gramática y ortografía, y el alumno que termina su bachillerato tiene dificulta al expresarse por escrito. Como docentes podemos apreciar diversas fallas en el escrito. Sin embargo, no podemos seguir buscando culpables. Ni desanimarnos o rendirnos por creer que no tiene solución. Al contrario, hay que partir de las dificultades que presenta el alumno y así guiarlo y enseñarle a superar su debilidad. La tarea del docente es persuadirlos de que poseen la capacidad de emplear esos conocimientos y habilidades (de aprenderlas si no las dominan) y con actitud positiva mostrarles que son capaces de mejorar su expresión escrita.

Es así que existen una serie de estudios sobre cómo se aprende a escribir. Esto ha dado lugar a una diversidad de modelos de enseñanza-aprendizaje de la expresión escrita, los cuales han servido de base para que los docentes podamos echar mano y ayudar a nuestros alumnos. Algunos de estos modelos responden a los procesos cognitivos del ser humano.

Uno de más empleados es el modelo de etapas propuesto por Rohman y Wlecke. Este consiste básicamente en tres etapas: pre-escritura (la invención), escritura y re-escritura. La tarea del docente será instruir en "las técnicas adecuadas que corresponden a las características de las tareas propias de cada etapa: cómo generar ideas, cómo combinar frases, seleccionar palabras, etc., cómo revisar el texto producido, etc." (Camps, 1989, p. 4). Como vemos, en este modelo el maestro instruye casi mecánicamente al estudiante sobre cómo debe escribir olvidando un poco que el proceso de escritura es individual.

De lo anterior se desprende el modelo cognitivo propuesto por Hayes y Flower. Según Camps (1989) este modelo tomaba en cuenta la relación entre "la memoria a largo plazo del escritor y el contexto de producción (...) y el proceso propiamente dicho" (p.5). El proceso de composición de este modelo es similar al propuesto por Rohman y Wlecke: planificación, textualización y revisión. La diferencia está en lo que ellos llamaron recursividad. Por tanto, el papel del docente en este tipo de modelo es enseñarle al alumno la importancia de revisar una y otra vez el escrito en todas sus etapas.

Dentro del modelo cognitivo existe una variedad de enfoques. Uno de ellos es el propuesto por De Beaugrande (citado por Camps, 1989) al cual llamó interacción de estadios paralelos. 
Según este autor la producción textual es compleja y por tanto no se puede concebir un modelo rígido, porque "el proceso de escritura es abierto (...) el texto puede ser siempre reconsiderado" (Camps, 1989, p. 8). No se debe pensar solo en el producto final, sino que el contexto de la escritura. Debe existir una razón para escribir y a partir de ahí escoger la tipología textual adecuada. En este sentido, la labor del docente estriba en escoger una tarea de escritura que motive al estudiante a mejorar su estilo.

Pereira y Di Stefano (2007) nos ofrecen un enfoque basado en la interdisciplinariedad: Ia psicología social, el análisis del discurso, la sociología y la historia social de la lectura y la escritura. Ellas plantean que en este enfoque la escritura debe ser vista como una práctica social, por tanto "el aprendizaje se produce a través de un proceso colaborativo, de interacción entre docentes y aprendices que van internalizando paulatinamente los conocimientos y habilidades que son objeto de enseñanza hasta alcanzar la autonomía" (p. 4). Podemos deducir que la labor del docente es primordial en la enseñanza aprendizaje de la expresión escrita. El docente debe dotar al alumno no solo de estrategias sino que debe compartir sus conocimientos y habilidades que como escritor experto domina y que el estudiante puede aprender.

Lo anterior es también compartido en el enfoque funcionalista en el cual se considera que el texto escrito toma significado en un contexto situacional concreto. Por tanto, la enseñanza-aprendizaje de la expresión escrita debe ser "en contacto con modelos textuales concretos y con las dificultades específicas que plantea su escritura" (Iglesias, 2010, p.8) Por tanto el maestro debe pensar en una situación real que amerite el empleo de una determinada tipología textual y enseñar a escribir ese texto. Para Iglesias (2010) las derivaciones pedagógicas de este enfoque son diversas:

1. El conocimiento y empleo en el aula de una gran variedad de textos reales, con tipologías y funciones comunicativas diversas.

2. La atribución de un significado social a la actividad de escritura, creando un contexto comunicativo real con propósitos y receptores concretos para el texto producido.

3. Énfasis especial en las necesidades comunicativas del alumno (Cassany, 1990). El enfoque funcional asume que cada alumno tiene necesidades de comunicación variadas, por lo que requerirá el aprendizaje de funciones y recursos lingüísticos distintos a los que podrá precisar otro compañero. Por ello, el docente tiene que proporcionar las ayudas, las estimulaciones y motivaciones que requiera el alumno en cada caso concreto.

4. El planteamiento de actividades de comunicación escrita, globales, reales y completas de forma similar a como se producen en los contextos comunicativos cotidianos. (p. 8)

Como hemos visto en esta breve reseña de modelos y enfoques sobre la enseñanza-aprendizaje de la expresión escrita, dos son los aspectos que podemos inferir. En primer lugar, que el proceso de escritura para que sea realmente efectivo, se debe plantear en función de un contexto real o verosímil. De esta forma el alumno se motiva y comprenderá que la escritura es una forma de comunicación la cual debe dominar. En segundo lugar, la función del docente no es solo de instruir, su guía debe estar acompañada de la demostración. El maestro al compartir sus experiencias escriturales le muestra al alumno las dificultades a las cuales se puede enfrentar y cómo puede superarlas. 


\section{El escritor-docente como modelo}

"En torno a la escritura sigue existiendo una concepción decimonónica: la capacidad de escribir es algo reservado tan sólo a algunos elegidos, los escritores" (Polanco, s.f., párr.2). Esta idea sigue perdurando en la mente de muchas personas. Y es posible que esta sea la causante de que muchos se resistan a mejorar su redacción. Sin embargo, la capacidad de escribir la tenemos todos sin excepción. Durante nuestros años de estudios vamos adquiriendo los conocimientos y habilidades necesarias para desarrollar esa capacidad.

Otra verdad latente es que los docentes son considerados como las personas idóneas para la escritura. Son el modelo a seguir durante el proceso de enseñanza-aprendizaje. Cabría hacerse la pregunta: ¿cuántos docentes se presentan ante sus alumnos como ejemplos de escritores?

Cassany (1999) plantea en artículo publicado en La revista para maestros y padres, un decálogo didáctico de la enseñanza de la composición. Esta es una serie de recomendaciones, de las cuales nos centraremos en dos de ellas. Estos tienen relación directa con la práctica docente durante la enseñanza-aprendizaje de la expresión escrita. El primero de ellos plantea que "el docente escribe en el aula: en público, ante la clase, con el aprendiz..." Según Cassany el alumno aprenderá mejor si observa a su maestro ejemplificar las técnicas que éste emplea para producir un texto. Si deseo que mis alumnos aprendan a elaborar un bosquejo, lo aconsejable es que vean como yo (docente) me colocó frente a la pizarra y marcador en mano demuestro cómo de una palabra puedo elaborar una serie de ideas y que con sus intervenciones construimos el bosquejo.

Esta debería ser una práctica cotidiana, sin embargo no es así, sobre todo en una situación de enseñanza tradicionalista en la cual se considera al docente como infalible. Sin embargo, Cassany (1999) nos invita a no tener "miedo de escribir con los alumnos, de mostrar nuestras limitaciones -también- y nuestras necesidades -consultar el diccionario, revisar, etc." Cuando nos mostramos de esta forma, el alumno observa que el proceso de escritura no se da como la magia. Que según la tipología textual debemos enfrentarnos a determinadas dificultades y que la diferencia entre un escritor competente y uno ingenuo estriba en el empleo de técnicas y estrategias para superar los problemas escriturales.

Un segundo aspecto a destacar del decálogo corresponde a la siguiente afirmación: "El docente actúa como lector, colaborador, asesor, no como árbitro, juez o jefe" (Cassany, 1999). Centrémonos en la palabra colaborador. El docente perfectamente puede colaborar en el escrito del estudiante. No se trata de marcar o tachar lo que consideremos como docente está mal, sino de brindarle algunas ideas al estudiante para mejorar su escrito. Esto puede ser de forma oral o escribiendo en el escrito del alumno, dando hasta dos posibles opciones para que su texto sea más claro o coherente. O, como señala Cassany ofrecer explicaciones satisfactorias al autor (alumno) sobre su texto. Recordemos que el proceso de escritura es individual, por tanto no podemos pretender que el estudiante escriba al modo que el docente quiera.

\section{A escribir con el alumno}

A continuación se irá explicando paso a paso como se trabajó con el grupo de Informática educativa del turno Sabatino. 
El programa de Español General contiene como temática final el ensayo, por tanto los alumnos en cinco sábados tienen que elaborar su escrito. Por cuestiones de tiempo y espacio se presentará dos aspectos del proceso: la planificación del ensayo y la textualización de la introducción.

Los estudiantes leyeron la obra "El triángulo de la Chela", una serie de cuentos de la autora nicaragüense Ángela Saballos. Después de la lectura de la obra se orientó la redacción del ensayo. Antes, se les explicó toda la teórica del ensayo y lo referente al proceso de composición: planificación, textualización, revisión y corrección.

\section{Primera sesión de clase:}

Tradicionalmente, se le indica al alumno que presente el tema y lo delimite. Además se le pide presentar la idea central o tesis. Muchas veces esto no es comprendido por el alumno y por lo general falla, sobre todo si no tienen un ejemplo concreto. Si bien es cierto, que después de varios intentos el estudiante logra hacerlo más o menos bien, creo que es menos desgastante si se le ofrece un ejemplo concreto de lo que se le pide.

Por tanto, es viable presentar una propuesta. Como docentes que nos vean crear el tema ante ellos. Es así que durante la clase se les presenta la siguiente propuesta:

Tema general: La obra "El triángulo de la Chela" de Ángela Saballos

Tema delimitado: Los personajes femeninos de los cuentos "El triángulo de la Chela" Tesis: La autora del "El triángulo de la Chela" nos presenta a través de sus personajes femeninos una tipificación de la mujer nicaragüense.

Una vez que leen la propuesta, se les pide hagan las observaciones debidas. En general, manifestaron que estaba bastante bien, y que se comprendía lo que se iba a escribir. Sin embargo, había que realizar un par de observaciones a lo que se había escrito: el tema delimitado aún no estaba totalmente claro y le faltaba mayor relación con la tesis; además, trabajar con todos los personajes femeninos podía ser agotador o muy repetitivo. Por tanto había que precisar en qué cuentos y aclarar el tema delimitado según la tesis. Así que en clase se reelaboró la propuesta:

Tema general: La obra "El triángulo de la Chela" de Ángela Saballos

Tema delimitado: Tipificación de la mujer nicaragüense en los personajes femeninos de los cuentos "Soledad Miranda", "Ya no sé ni quién soy" y "María Te"

Tesis: La autora del "El triángulo de la Chela" nos presenta a través de sus personajes femeninos una tipificación de la mujer nicaragüense.

Los estudiantes pudieron apreciar cómo se delimitó el tema y cómo se relacionó con la tesis. Posteriormente, decidí esbozar a grandes rasgos lo que sería el bosquejo del ensayo. Debe aclararse que las ideas que se van a plasmar pertenecerán al desarrollo del ensayo y no a la introducción. Así que se procedió a escribir en la pizarra una serie de ideas sobre lo que se pretendía escribir, luego se ordenan. 
Revista de Lengua y Literatura. Vol.4, núm. 2, enero-junio 2018

Tabla 1.

Primer planteamiento de las ideas

\begin{tabular}{l|l|}
\hline Primeras ideas & \multicolumn{1}{|c|}{ Ideas ordenadas } \\
\hline La autora & $\begin{array}{l}\text { 1. Ángela Saballos autora de la } \\
\text { obra "El triángulo de la Chela" }\end{array}$ \\
La mujer nicaragüense & 2. Rasgos de la mujer nicara- \\
Rasgos de la mujer & güense. \\
Los personajes de los cuentos & 3. Tipificación de la mujer según \\
María Te & $\begin{array}{l}\text { los personajes de los cuentos } \\
\text { La maestra }\end{array}$ \\
$\begin{array}{l}\text { Soledad } \\
\text { Tipos de mujer realidad de la mujer nica- } \\
\text { Realidad presentada en los los cuentos. } \\
\text { cuentos }\end{array}$ & \\
\hline
\end{tabular}

Al finalizar la sesión de clase, se les pide a los alumnos que trabajen en parejas y definan su tema y tesis. En la próxima sesión de clase lo presentaran en clase.

\section{Segunda sesión}

Al iniciar la sesión de clase se les pide a los alumnos colocar sus propuestas (traídas en papelógrafos) en la pizarra y pared de la sección. Esto contribuye a que se realice una coevaluación de cada una de las propuestas. Por razones de espacio, solo se presentan dos propuestas, las cuales se han transcritos tal y como lo presentaron los alumnos.

\section{Equipo 1:}

Tema: Elementos históricos sociales y culturales presentes en los cuentos del triángulo de las chelas.

Tema delimitado: Elementos históricos, sociales y culturales en los cuentos: De cristo, de psiquiatría y de la cartomancia, I want to hold your hand y ya no sé ni quién soy.

Idea central: Los elementos históricos, sociales y culturales de los cuentos se encuentran presentes en la vida actual de la población Nicaragüense haciendo énfasis en nuestra realidad.

Propósito: Concientizar a las personas como los elementos históricos, sociales y culturales están presentes en la vida actual afectando a toda la población Nicaragüense sin importar sexo, raza o posición económica.

Explicar los elementos históricos, sociales y culturales presentes en la vida actual afectando a la población Nicaragüense sin importar sexo, raza o posición económica. 


\section{Equipo 2:}

Tema general: Los personajes de los cuentos de "El triángulo de la chela"

Tema delimitado: La visión negativa, masculina en los cuentos "Ya no sé ni quién soy", "Ladrón que roba a ladrón", "El retrato".

Idea central: Dichos personajes de estos cuentos tienen una similitud al hombre real. Nuestra autora atrevez de estos personajes masculino nos otorga una estrecha relación, entre sus personajes y el hombre real. Ya que en dichos casos ambos hombres son en su mayoría iguales.

Propósito: Hacer un enfoque y exponer el porque nuestra autora refleja a los personajes masculino de esta manera, con el hombre real.

Como podemos observar, los escritos presentaron algunos problemas de redacción. Durante la sesión de clase se hicieron las observaciones necesarias. Se les pidió corrigieran la ortografía y los aspectos de coherencia y cohesión de los textos. Por ejemplo, al equipo 1 tenía que valorar escoger uno de los tres aspectos que proponían; explicar con mayor claridad la idea central; además, fusionar los propósitos porque hay repetición. En el caso del equipo 2, se les sugirió precisar la idea central; y expresar con más claridad el propósito.

Luego de las sugerencias, se les pidió realizar las correcciones debidas y mostrarlas nuevamente.

\section{Equipo 1}

Tema: Elementos históricos sociales y culturales presentes en los cuentos del triángulo de las chelas.

Tema delimitado: Elementos socio-culturales en los cuentos: "De Cristo, de psiquiatría y de la cartomancia", "I want to hold your hand" y "Ya no sé ni quién soy".

Idea central: Los elementos socio-culturales que se encuentran presentes en los cuentos son parte de la realidad nicaragüense.

Propósito: Explicar los elementos socio-culturales presentes en los cuentos y su relación con la vida actual. 


\section{Equipo 2}

Tema general: Los personajes de los cuentos de "El triángulo de la chela"

Tema delimitado: La visión negativa de los personajes masculinos en los cuentos "Ya no sé ni quién soy", "Ladrón que roba a ladrón", "El retrato".

Idea central: La autora a través de los personajes masculinos nos presenta una similitud con los rasgos del machismo.

Propósito: Exponer los puntos negativos del machismo en los cuento.

\section{Tercera sesión}

En esta sesión, se les explica la importancia de la introducción del ensayo y los elementos que deben estar presentes: explicación de la temática, explicitación de la idea central y el propósito del ensayo. Posteriormente, se escribe un ejemplo de introducción:

Tema general: La obra "El triángulo de la Chela" de Ángela Saballos

Tema delimitado: Tipificación de la mujer nicaragüense en los personajes femeninos de los cuentos "Soledad Miranda", "Ya no sé ni quién soy" y "María Te"

Tesis: La autora del "El triángulo de la Chela" nos presenta a través de sus personajes femeninos una tipificación de la mujer nicaragüense.

Leer los cuentos de Ángela Saballos ha sido toda una experiencia. En cada uno de sus cuentos logra mostrarnos una diversidad de personajes que a través de sus vivencias podemos conocerlos y conocernos un poco más, sobre todo a las mujeres nicaragüenses. Decimos esto porque la autora nos presenta a través de los personajes femeninos una tipificación de la mujer nicaragüense. Por tanto, en este ensayo pretendemos explicar cómo cada una de las protagonistas de los cuentos, representan a las nicaragüenses.

El ejemplo es sometido a revisión, de esta forma el alumno puede comprender que no siempre la primera versión es la mejor. Lo primero que se debe revisar es si los elementos planteados en la planificación se encuentran en el párrafo de introducción. Luego, observar si no hay repeticiones, si los elementos de enlace son los más idóneos para la cohesión del texto, si se logra atrapar la atención del lector, entre otros detalles, se procede a reescribir la introducción.

Luego de esto, se procede a realizar la corrección en clase, siempre pidiendo la sugerencia de los alumnos. He aquí la nueva propuesta: 
Los cuentos reunidos en la obra "El triángulo de la Chela" en su mayoría son relatos personificados por mujeres. Son pocas las obras nicaragüense que abordan la problemática y vida de la mujer. Su forma de pensar y sentir lo podemos ir leyendo en cada uno de los cuentos. Mujeres que luchan, aman, sueñan, ríen, lloran, sufren y tienen esperanzas.

Por tanto, leer los cuentos de Ángela Saballos ha sido toda una experiencia. Sobre todo los relatos: "Soledad Miranda", "Ya no sé ni quién soy" y "María Te". Sus protagonistas a través de sus vivencias podemos conocerlos y conocernos un poco más, sobre todo a las mujeres. Decimos esto porque Saballos nos presenta a través de los personajes femeninos una tipificación de la mujer nicaragüense. Por tanto, en este ensayo pretendemos explicar cómo cada una de las protagonistas de los cuentos, representan a las nicaragüenses.

Luego, se les pide a los alumnos escribir su propia introducción a partir de lo expresado en la planificación. A medida que ellos van escribiendo su introducción, se le pide ir mostrando los avances. Al final de la sesión los entregan para su revisión. He aquí los resultados:

\section{Equipo 1}

Los cuentos del triángulo de la chela: De cristo, de psiquiatría y de la cartomancia; I want to hold your hand y ya no sé quién soy contienen elementos socio-culturales que se encuentran presentes en la vida actual, haciendo énfasis en la realidad de nuestra población nicaragüense. Estos elementos son el machismo, el racismo y la envidia que muchas veces adquieren las personas como cultura histórica o herencia de sus antepasados por eso queremos concientizar a las personas como estos elementos históricos, sociales y culturales afectan a todos sin importar el sexo, raza o posición económica.

\section{Equipo 2}

¿Cómo considera usted a la mayoría de los hombres? Hay diferentes opiniones y formas de considerarlos, unos lo consideran que son: ambles, cariñosos, buenos, etc. Otros lo consideran de forma muy distinta o mejor dicho todo lo contrario, en el siguiente ensayo veremos la negatividad del hombre, los personajes de los cuentos de Ángela Saballos presentan una similitud con los rasgos del machismo, estos casos lo veremos en los siguientes cuentos: ya no se ni quien soy, Ladrón que roba a ladrón y el retrato. 
En la siguiente sesión de clase se les entrega su trabajo con la revisión y sugerencias realizadas. Los alumnos deben corregir y se continúa con el desarrollo y conclusión del ensayo. Por razones de espacio, solo presentamos las introducciones presentadas en el ensayo final.

¿Cómo considera usted a la mayoría de los hombres? Hay diferentes opiniones y formas de considerarlos, unos lo consideran que son: ambles, cariñosos, buenos, afectuosos, etc. Otros lo consideran de forma muy distinta o mejor dicho todo lo contrario. En nuestro siguiente ensayo veremos los aspectos negativos del hombre con los personajes de los cuentos de Ángela Saballos. Ya que estos presentan una similitud con los rasgos del machismo. Estos casos lo veremos en los siguientes cuentos del libro "El triángulo de la Chela": Ya no sé ni quién soy, Ladrón que roba a ladrón y el retrato.

Enseñar a redactar no es una tarea fácil. Sin embargo, como docentes necesitamos implementar estrategias que nos permitan orientar a cada uno de los alumnos. Escribir con ellos es una forma dinámica de compartir con el alumno el proceso de enseñanza-aprendizaje de la expresión escrita.

Los estudiantes al observar como escribe el maestro tiene una idea de lo que él puede realizar. Su aprendizaje es más significativo, ya que ven como las ideas se originan de algunas palabras y luego se transforman en párrafos.

Una desventaja de esta forma de trabajo es el tiempo. Desgraciadamente, se invierte mucho tiempo y por eso no puede darse un párrafo de introducción, por ejemplo, totalmente acabado y correcto. Siempre se presentan algunas fallas o errores. El tiempo destinado a la asignatura de Español General en la universidad no es suficiente para tratar de ayudar al alumno a superar los vacíos que trae consigo.

Por último, una asignación de escritura bien orientada, ayuda al estudiante para superar sus errores. Por tanto, no es solo responsabilidad del docente de Español velar porque el alumno supere sus problemas de redacción. 


\section{$\longrightarrow$ REFERENCIAS}

Camps, A. (1989) Modelos del proceso de redacción: algunas implicaciones para la enseñanza. Infancia y aprendizaje. 49,3-19.

Cassany, D. (1999) Alegría de enseñar. La revista para maestros y padres. 40, 22-28. Colombia.

Cassany, D. (2002) La cocina de la escritura. 11 ed. Anagrama: Barcelona.

Iglesias, G. (2010) Enfoques didácticos de enseñanza de la redacción y composición. Revista Intercambio. Consultada en unb.revistaintercambio.net.br

Pereira, C y Stefano, M. di (2007) El taller de escritura en posgrado: representaciones sociales e interacción entre pares. Revista signos. v.40 n.64 Valparaíso versión on-line.

Polanco, J (1999) Enseñar a escribir. Compartir los riesgos de la escritura. Revista Textos de didáctica de la lengua y la literatura. 21, 101-109. 